\title{
S1 Dataset
}

\section{Recombinant Tc80 amino acid sequence}

MRSVYPLARRSMAAYTMHNMTVPEPYDYLEDPENPETKTFVNEQNAFFEEYFASEAELRKKIFESISNSQDYPRTSN PSYINGHYYYYHNSGLQNQSVLMRAMSLTDTAPSIFLDPNSMSSDGTTALKATAWSEDESMLAYSLSDKGSDWQR IHVRRADTVEDTSDVIEWAKFTAIAWWHNLGFFYTRYPALQGDVDKGAETDAAQDAFICFHRIGRPQDEDVVILSV PEHPQWNMGASVSDCHSYVIVVLFDGCEPHNLVWVAELPSVEKGLGSEPLVFKKLVNEFAGRYTYLGNEGSTFYFV TTRDAPRKKIVSIDIHTGQETVIVEQQRSVLSQAALVKKTLLLAYLEDVKDVFYYCRLEDPTLNAIPLPIGTITSFFSDRK KDFVSFKITSFLLPGRSFFLDINDPQSSLRVFKDDTVEGLLVDDFVTEQTFYNSSDGVRIPMFIVYRKGSVSSESPLLLY GYGGFNIPLTPAFSSSRMVFLRDLGGVLAVLNIRGGGEYGEEWHDAGRRACKQNCFTDFIEGAKFLHRQGYGSPQ TTAIMGGSNGGLLVAAVANQAPELFRCVVCRVGVLDMYKFHKFTIGHAWKSDYGDPEKEEDFRVLQQYSPLHNIK SGIKYPAILVVTGDHDDRVVPLHSLKYVATLQHMNPNEGGPFLARIEVAAGHGAGKPTSKILREAGDIYTFIAKNINA SWKEHHHHHH

Mouse MHC-I H-2Kk nonapeptide prediction results

Top 10 SYFPEITHI results

(http://www.syfpeithi.de/bin/MHCServer.dll/EpitopePrediction.htm)

\begin{tabular}{|c|c|c|}
\hline Pos & 123456789 & Score \\
\hline 54 & SEAELRKKI & 25 \\
\hline 362 & LEDPTLNA I & 22 \\
\hline 419 & DDTVEGLLV & 22 \\
\hline 487 & R D L G G V LAV & 22 \\
\hline 168 & IEWAKFTAI & 21 \\
\hline 220 & DEDVVILSV & 21 \\
\hline 202 & TDAAQDAFI & 19 \\
\hline 296 & NEGSTFYFV & 19 \\
\hline 307 & RDAPRKKIV & 19 \\
\hline 255 & CEPHNLVWV & 18 \\
\hline
\end{tabular}

Top 10 RANKPEP results (http://imed.med.ucm.es/Tools/rankpep.html)

\begin{tabular}{|c|c|c|c|c|c|c|c|}
\hline RANK & POS. & N & SEQUENCE & C & MW (Da) & SCORE & $\%$ OPT. \\
\hline 1 & 54 & YFA & SEAELRKKI & FES & 1055.25 & 22.861 & $43.57 \%$ \\
\hline 2 & 32 & LED & PENPETKTF & VNE & 1044.13 & 20.669 & $39.39 \%$ \\
\hline 3 & 422 & DDT & VEGLLVDDF & VTE & 988.11 & 19.956 & $38.03 \%$ \\
\hline 4 & 166 & DTS & DVIEWAKFT & AIA & 1067.24 & 12.933 & $24.65 \%$ \\
\hline 5 & 440 & YNS & SDGVRIPMF & IVY & 1003.19 & 12.468 & $23.76 \%$ \\
\hline 6 & 592 & WKS & DYGDPEKEE & DFR & 1063.06 & 11.921 & $22.72 \%$ \\
\hline 7 & 683 & AGD & IYTFIAKNI & NAS & 1064.29 & 10.947 & $20.86 \%$ \\
\hline 8 & 575 & VGV & LDMYKFHKF & TIG & 1210.46 & 10.705 & $20.40 \%$ \\
\hline 9 & 207 & AAQ & DAFICFHRI & GRP & 1103.32 & 10.57 & $20.14 \%$ \\
\hline 10 & 679 & ILR & EAGDIYTFI & AKN & 1010.12 & 10.466 & $19.95 \%$ \\
\hline
\end{tabular}


Top 10 IEDB results (http://tools.immuneepitope.org/mhci/)

\begin{tabular}{|c|c|c|c|c|c|}
\hline allele & start & end & length & peptide & percentile_rank \\
\hline $\mathrm{H}-2-\mathrm{Kk}$ & 54 & 62 & 9 & SEAELRKKI & 0.1 \\
\hline $\mathrm{H}-2-\mathrm{Kk}$ & 168 & 176 & 9 & IEWAKFTAI & 0.2 \\
\hline $\mathrm{H}-2-\mathrm{Kk}$ & 296 & 304 & 9 & NEGSTFYFV & 0.3 \\
\hline $\mathrm{H}-2-\mathrm{Kk}$ & 202 & 210 & 9 & TDAAQDAFI & 0.6 \\
\hline $\mathrm{H}-2-\mathrm{Kk}$ & 275 & 283 & 9 & SEPLVFKKL & 0.6 \\
\hline $\mathrm{H}-2-\mathrm{Kk}$ & 362 & 370 & 9 & LEDPTLNAI & 0.6 \\
\hline $\mathrm{H}-2-\mathrm{Kk}$ & 42 & 50 & 9 & NEQNAFFEE & 1.1 \\
\hline $\mathrm{H}-2-\mathrm{Kk}$ & 458 & 466 & 9 & SESPLLLYG & 1.2 \\
\hline $\mathrm{H}-2-\mathrm{Kk}$ & 432 & 440 & 9 & TEQTFYNSS & 1.6 \\
\hline
\end{tabular}

